\title{
A DIRECTION OF BIFURCATION FORMULA IN THE THEORY OF THE IMMUNE RESPONSE ${ }^{1}$
}

BY GEORGE H. PIMBLEY, JR.

Communicated by A. H. Householder, September 7, 1975

In previous work [1], I derived by biological reasoning and mathematical reduction the following system, attributable to G. I. Bell:

$$
\begin{gathered}
d u / d s=u\left[\lambda_{1}+k \lambda_{1} u-k\left(\alpha_{1}-\lambda_{1}\right) v+k n \lambda_{1} w\right] \\
d v / d s=\beta\left\{v\left[-\lambda_{2}-k\left(\alpha_{2}+\lambda_{2}\right) u-k \lambda_{2} v-k n \lambda_{2} w\right]+k \gamma u w\right\} \\
d w / d s=w\left[-\lambda_{3}+k\left(\alpha_{3}-\lambda_{3}\right) u-k \lambda_{3} v-k n \lambda_{3} w-\left(k \alpha_{3} / \theta\right) u w\right]
\end{gathered}
$$

Equations (1) simulate the immune response of an organism to antigen invasion. The dependent variables $u, v, w$ are, respectively the concentrations of antigens, antibodies, and antibody-producing cells. The meanings of all parameters and constants are found in [1, pp. 93-96].

Equations (1) have two nontrivial rest points. The one nearest the origin, $\left(u_{f}, v_{f}, w_{f}\right)$, is stable or unstable according to whether $\beta>\beta_{c}$ or $\beta<\beta_{c}$, where $\beta_{c}>0$ is a critical value of the parameter $\beta$ in equation (1b). It is shown [1, Theorem 1] that at $\beta=\beta_{c}$, a continuous family of periodic solutions bifurcates from $\left(u_{f}, v_{f}, w_{f}\right)$. I was able to obtain a direction of bifurcation formula only in the special case where $\lambda_{3}=0$. Namely, periodic solutions bifurcate to the left (right) of $\beta_{c}$, and are stable (unstable) if

$$
\beta_{c}>\left(\alpha_{1}-\lambda_{1}\right) \lambda_{1} /\left(\left(\alpha_{1}-\lambda_{1}\right)\left(\alpha_{2}+\lambda_{2}\right)+2 \lambda_{1} \lambda_{2}\right), \quad(<) .
$$

Herein I announce the development of a general formula for direction of bifurcation in equations (1), which approaches condition (2) as $\lambda_{3} \rightarrow 0$. An analytic direction of bifurcation formula will be important in developing the global theory of these bifurcated families of periodic solutions, and in ascribing possible biomedical implications. I describe the new formula.

First we substitute $u=u_{f}+u^{0}, v=v_{f}+v^{0}, w=w_{f}+w^{0}$ into equations (1), and thus obtain equations centered at $\left(u_{f}, v_{f}, w_{f}\right)$. Then we let $A_{\beta_{c}}$ be the matrix of the linear part of these centered DE's, with $\beta=\beta_{c}$. The matrix $A_{\beta_{c}}$ has the three linearly independent eigenvectors represented symbolically as

$$
\left(\xi_{1}, \eta_{1}, \zeta_{1}\right), \quad\left(\bar{\xi}_{1}, \bar{\eta}_{1}, \bar{\zeta}_{1}\right), \quad(\xi, \eta, \zeta)
$$

AMS (MOS) subject classifications (1970). Primary 92A05; Secondary 34C05.

1 Work performed under the auspices of the U. S. Energy Research and Development Administration. 
Equations (1) also have an invariant surface passing through $\left(u_{f}, v_{f}, w_{f}\right)$, represented as follows:

$$
z=\phi(x, y)=a_{20} x^{2}+a_{11} x y+a_{02} y^{2}+o\left(x^{2}+y^{2}\right),
$$

where $x, y, z$ are new dependent variables obtained from $\left(u^{0}, v^{0}, w^{0}\right)$ through a principal axis transformation.

We must define the following quantities:

$$
\begin{aligned}
& C=i s_{12}^{-1} \beta_{c} \lambda_{2}, \quad D=i s_{12}^{-1} \beta_{c}\left(\alpha_{2}+\lambda_{2}\right)+i s_{11}^{-1}\left(\alpha_{1}-\lambda_{1}\right), \\
& E=i s_{12}^{-1} \beta_{c} \lambda_{2} n+i s_{13}^{-1} \lambda_{3}, \\
& F=-i s_{12}^{-1} \beta_{c} \gamma-i s_{11}^{-1} \lambda_{1} n-s_{13}^{-1}\left(\alpha_{3}-\lambda_{3}-\left(2 \alpha_{3} / \theta\right) w_{f}\right), \\
& G=-i s_{11}^{-1} \lambda_{1}, \quad H=i s_{13}^{-1}\left(n \lambda_{3}+\left(\alpha_{3} / \theta\right) v_{f}\right), \quad I=i s_{13}^{-1} \alpha_{3} / \theta,
\end{aligned}
$$

where

$$
s_{11}^{-1}=\frac{\zeta \bar{\eta}_{1}-\bar{\zeta}_{1} \eta}{\Delta}, \quad s_{12}^{-1}=\frac{-\zeta \bar{\xi}_{1}+\bar{\zeta}_{1} \xi}{\Delta}, \quad s_{13}^{-1}=\frac{\eta \bar{\xi}_{1}-\bar{\eta}_{1} \xi}{\Delta}
$$

with

$$
\Delta=2 i\left[\xi \operatorname{Im}\left(\eta_{1} \bar{\zeta}_{1}\right)-\eta \operatorname{Im}\left(\xi_{1} \bar{\zeta}_{1}\right)+\zeta_{1} \operatorname{Im}\left(\xi_{1} \bar{\eta}_{1}\right)\right]
$$

We make the realistic assumption that $\alpha_{1}>\lambda_{1}, \alpha_{3}>\lambda_{3}$.

Also we need the constant $l=\sqrt{\operatorname{trace} A_{\beta_{c}}^{c}}$ where $A_{\beta_{c}}^{c}$ is the first compound of the matrix $A_{\beta_{c}}$.

Using the constants defined in (4), I put forward the following direction of bifurcation criterion: Define

$$
\begin{aligned}
& \kappa=\frac{1}{l} \operatorname{Re}\left\{i ( a _ { 2 0 } - a _ { 0 2 } + i a _ { 1 1 } ) \left[2 \eta \bar{\eta}_{1} C+\left(\xi \bar{\eta}_{1}+\eta \bar{\xi}_{1}\right) D+\left(\eta \bar{\zeta}_{1}+\zeta \bar{\eta}_{1}\right) E\right.\right. \\
& \left.+\left(\xi \bar{\zeta}_{1}+\zeta \bar{\xi}_{1}\right) F+2 \xi \bar{\xi}_{1} G+2 \zeta \bar{\zeta}_{1} H\right] \\
& +2 i\left(a_{20}+a_{02}\right)\left[2 \eta \eta_{1} C+\left(\xi \eta_{1}+\eta \xi_{1}\right) D+\left(\eta \zeta_{1}+\zeta \eta_{1}\right) E\right. \\
& \left.+\left(\xi \zeta_{1}+\zeta \xi_{1}\right) F+2 \xi \xi_{1} G+2 \zeta \zeta_{1} H\right] \\
& \left.+i \xi_{1} \zeta_{1}^{2} I+2 i \xi_{1}\left|\zeta_{1}\right|^{2} I\right\} \\
& +\frac{2}{l^{2}} \operatorname{Re}\left\{-(i / 2)\left[2\left|\eta_{1}\right|^{2} \bar{C}+\left(\xi_{1} \bar{\eta}_{1}+\bar{\xi}_{1} \eta_{1}\right) \bar{D}+\left(\eta_{1} \bar{\zeta}_{1}+\bar{\eta}_{1} \xi_{1}\right) \bar{E}\right.\right. \\
& \left.+\left(\xi_{1} \bar{\zeta}_{1}+\bar{\xi}_{1} \zeta_{1}\right) \bar{F}+2\left|\xi_{1}\right|^{2} \bar{G}+2\left|\zeta_{1}\right|^{2} H\right] \\
& \left.\times\left[\bar{\eta}_{1}^{2} \bar{C}+\bar{\xi}_{1} \bar{\eta}_{1} \bar{D}+\bar{\eta}_{1} \bar{\zeta}_{1} \bar{E}+\bar{\xi}_{1} \bar{\zeta}_{1} \bar{F}+\xi_{1}^{2} \bar{G}+\bar{\xi}_{1}^{2} \bar{H}\right]\right\} \text {. }
\end{aligned}
$$

The criterion is as follows: If $\kappa$ is negative (positive), then the bifurcation periodic solutions of equations (1) exist in a left (right) neighborhood of $\beta=\beta_{c}$, and are stable (unstable). 
As can be seen in (5), the quantities $C, D, E, F$ are linear in the critical value $\beta_{c}$. Therefore formula (6) gives a representation that is a quadratic function of $\beta_{c}$. Moreover it turns out that the quadratic equation $\kappa=0$ has a largest positive root when $\lambda_{3} \geqslant 0$. We call this root $\beta_{c c}$.

Then the criterion for direction of bifurcation can be interpreted as follows: If $\beta_{c}>\beta_{c c},(<)$, the bifurcated periodic solutions emanating from $\beta=\beta_{c}$ exist in a left (right) interval of $\beta_{c}$ and are stable (unstable).

The quantity on the right in inequality (2) is, in fact, the greatest positive root of $\kappa=0$ when $\lambda_{3}=0$.

The proof utilizes the focal point-saddle point type of analysis that goes back to Poincaré [2, pp. 167-181].

\section{REFERENCES}

1. G. H. Pimbley, Jr., Periodic solutions of third order predator-prey equations simulating an immune response, Arch. Rational Mech. Anal. 55 (1974), 93-123.

2. H. Poincaré, Collected works. Vol. I, Gauthier-Villars, Paris, 1951.

THEORETICAL DIVISION, UNIVERSITY OF CALIFORNIA, LOS ALAMOS SCIENTIFIC LABORATORY, LOS ALAMOS, NEW MEXICO 87545 